\title{
Polarimetric DBR fiber laser sensor for strain-temperature discrimination
}

\author{
L. Rodriguez-Cobo*, M.A. Quintela, I. Laarossi and J.M. Lopez-Higuera \\ Photonics Engineering Group, University of Cantabria, 39005, Santander (Spain)
}

\begin{abstract}
A polarimetric Distributed Bragg Reflector (DBR) laser sensor in a low birefringent Er-doped fiber has been proposed. The spectral overlap of two uniform fiber Bragg gratings (FBG) has been employed as filtering technique to achieve a Single Longitudinal Mode (SLM) regime. By measuring the RF beat frequency between the two orthogonal polarized lasing modes and the absolute wavelength of one mode, both strain and temperature has been determined simultaneously.
\end{abstract}

Keywords: Fiber Bragg grating (FBG), erbium doped fiber, fiber laser, strain and temperature sensor

\section{INTRODUCTION}

Multi-parameter sensors can solve the cross sensitivity problem (e.g. FBGs) when working in real scenarios and also reduce the complexity, size and cost of the whole sensing system. Many techniques based on fiber Bragg gratings (FBG) have been reported for multi-parameters measurement, such as embedded FBG [1], different types of FBG [2], superstructure Bragg grating [3], combination with a long period grating [4]. However, for remote sensing applications, the FBG sensors can be limited by their signal-to-noise ratio (SNR) and, consequently, reducing the accuracy of the sensor system. Active fiber grating sensors, (e.g sensors where gratings form part of a fiber laser) have been proved to be a reliable technology to overcome this limitation due to their narrow linewidth and high output power.

Fiber lasers based on Distributed Bragg Reflector (DBR) or Distributed Feedback laser (DFB) have been employed as polarimetric sensors for simultaneous measurement of strain and temperature [5], [6]. These fiber lasers typically exhibit two orthogonal polarization modes caused by the fiber birefringence. Any external perturbation such as strain or temperature, changes the birefringence and, therefore, the polarization beat frequency. Moreover, fiber lasers should operate in single longitudinal mode regime (SLM) to achieve high resolutions in sensing systems. For SLM regime, usually DBR fiber laser may not be longer than a few centimeters and the grating bandwidth has to be below $\sim 0.2 \mathrm{~nm}$. Nevertheless, narrow filtering technique based on the spectral overlapping of two uniform FBGs [7] has been proved as a reliable technique to achieve a SLM operation in DBR fiber lasers, even when long cavity lengths are required.

In this work, a DBR laser sensor working on the Single Longitudinal Mode regime has been employed to discriminate between two measurements (strain and temperature). The DBR laser structure has two orthogonal polarization modes whose frequency distance exhibits different sensitivities to strain and temperature variations than the FBGs, allowing the simultaneous measuring of both parameters. The experimental results based on a proof-of-concept laser enable this technique to be employed in structural applications.

\section{WORKING PRINCIPLE}

The DBR fiber laser sensor consist of two uniform FBGs written in an erbium doped fiber with an appropriate distance between them. The spectral overlap technique has been used to achieve single longitudinal mode (SLM) laser [7]. This method is based on combining two uniform FBGs of matched Bragg wavelengths, being one slightly detuned and keeping its reflection spectra partially overlapped with the other. In this way, the whole spectral overlapped response of both FBGs becomes narrower than the ones associated with the two individual FBGs. Moreover, due to the fiber birefringence introduced during the fiber fabrication and/or the grating inscription, there are two orthogonal polarization modes and, consequently, the laser emits in two different lasing wavelengths. For a low birefringent fiber $\left(\mathrm{n}_{\mathrm{x}} \approx \mathrm{n}_{\mathrm{y}} \approx \mathrm{n}\right.$ where $\mathrm{n}_{\mathrm{x}, \mathrm{y}}$ are the modal refractive indexes), the beat frequency is given by:

*luis.rodriguez@unican.es; phone +34 942200877; fax +34 942200877

23rd International Conference on Optical Fibre Sensors, edited by José Miguel López-Higuera,

Julian Jones, Manuel López-Amo, José Luis Santos, Proc. of SPIE Vol. 9157, 91572B

(C) 2014 SPIE $\cdot$ CCC code: $0277-786 X / 14 / \$ 18 \cdot$ doi: $10.1117 / 12.2059551$ 

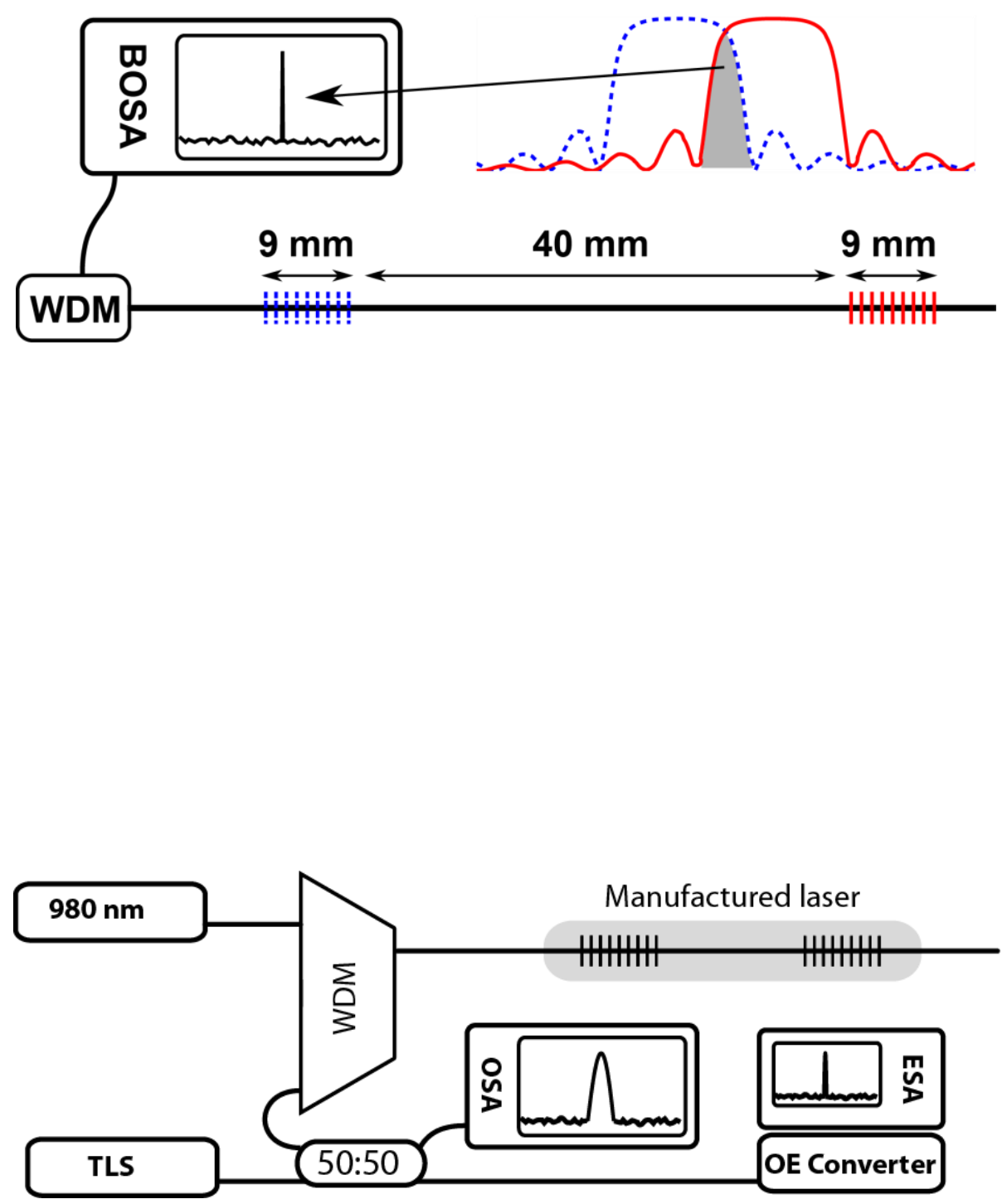

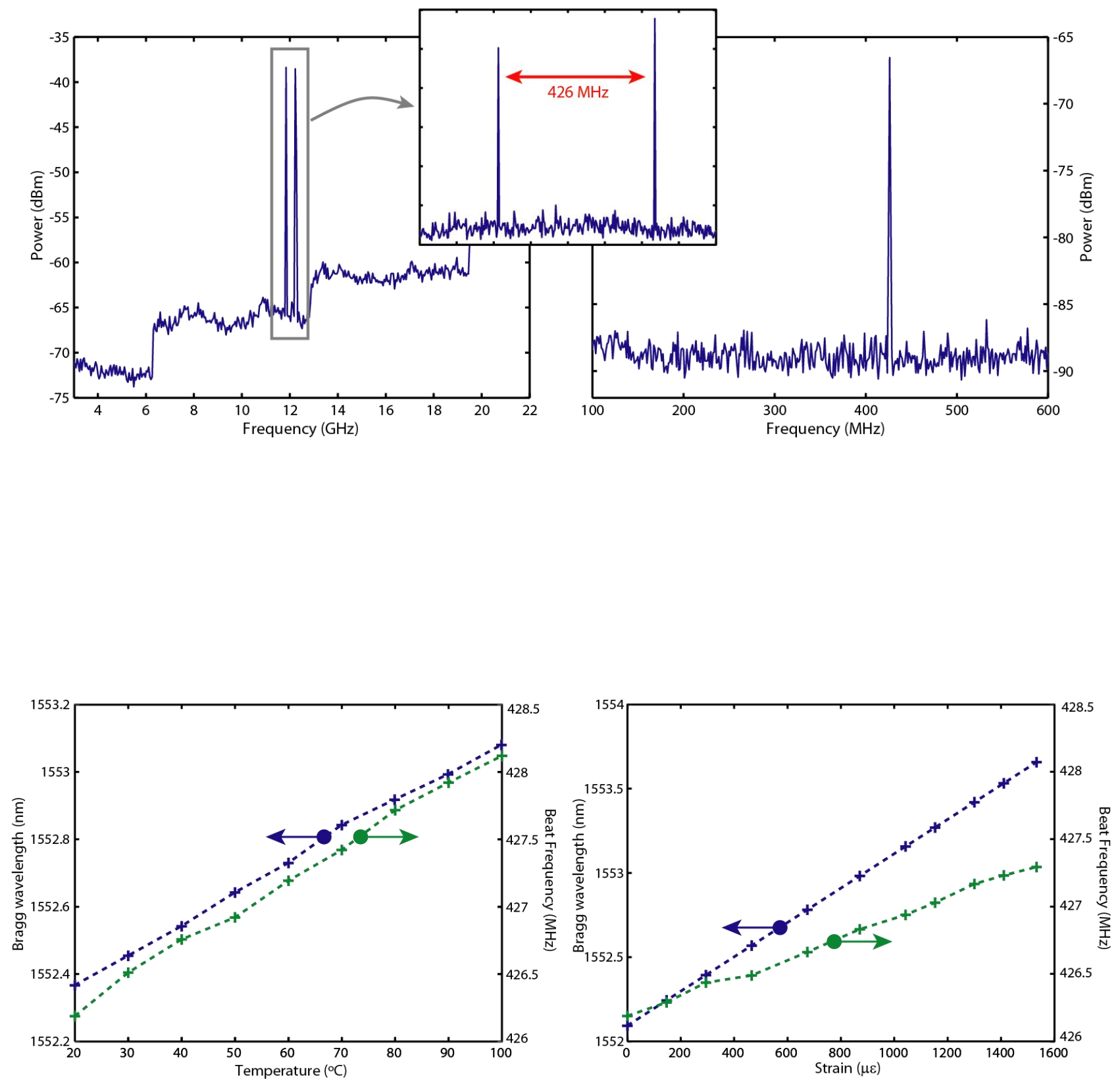


\subsection{Error analysis}

Both sensitivities (optical and electrical) are different, thus, consequently, it is possible to obtain both measurands simultaneously, by solving the system of equations [1]. In Fig. 5, the achieved resolution is depicted. The graph was obtained by varying one measure while the other was fixed. The applied sweeps (strain and temperature) are depicted (solid lines) against the computed variations using wavelength drifts (dots).

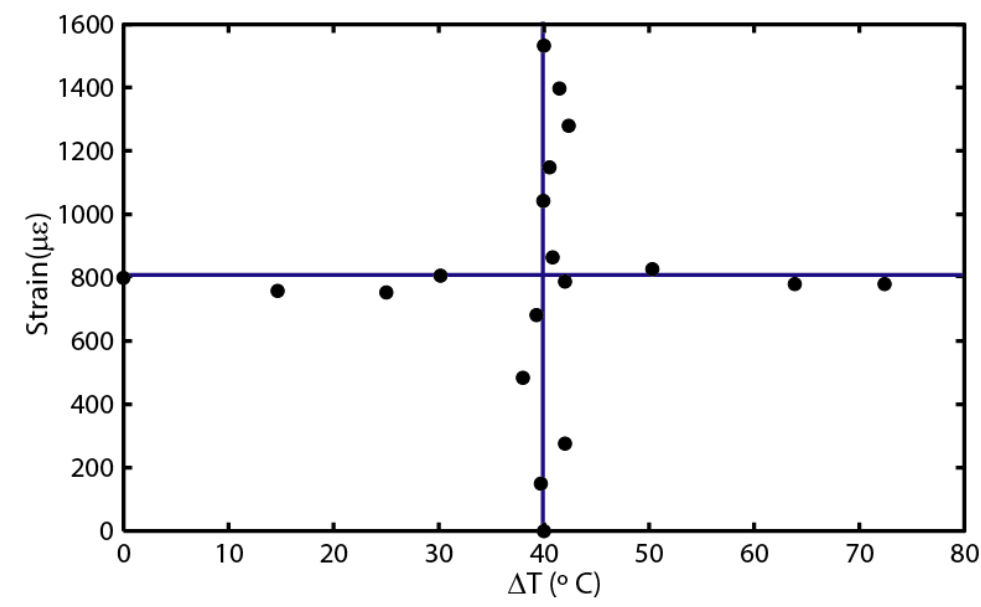

Figure 5. Resolution of the manufactured laser for different ramps.

The achieved error depends on the sensitivities difference, thus a better discrimination can be achieved by increasing this difference. For the manufactured laser there were high differences between sensitivities (different domains), achieving a mean strain error of $8.1 \mu \epsilon$, being the temperature mean error $2.1^{\circ} \mathrm{C}$ during the discrimination.

\section{CONCLUSIONS}

In this work, a dual polarization DBR fiber laser sensor has been designed to achieve simultaneous measurements of strain and temperature. Using the spectral overlapping technique in a commercial low birefringent Er-doped fiber, a proof-of-concept fiber laser sensor has been manufactured and experimentally tested. This laser operated in robust SLM regime for different working conditions and in two orthogonal polarization modes. The simultaneous measurement of strain and temperature has been experimentally demonstrated by measuring the laser wavelength and polarization beat frequency. This work has been supported by the project TEC2010-20224-C02-02 and grant AP2009-1403

\section{REFERENCES}

[1] L. Rodriguez-Cobo, A. Marques, J. López-Higuera et al., "New design for temperature-strain discrimination using fiber Bragg gratings embedded in laminated composites," Smart Materials and Structures, 22(10), 105011 (2013).

[2] X. Shu, Y. Liu, D. Zhao et al., "Dependence of temperature and strain coefficients on fiber grating type and its application to simultaneous temperature and strain measurement," Optics letters, 27(9), 701-703 (2002).

[3] B.-O. Guan, H.-Y. Tam, X.-M. Tao et al., "Simultaneous strain and temperature measurement using a superstructure fiber Bragg grating,” Photonics Technology Letters, IEEE, 12(6), 675-677 (2000).

[4] H. Patrick, G. Williams, A. Kersey et al., "Hybrid fiber Bragg grating/long period fiber grating sensor for strain/temperature discrimination,” Photonics Technology Letters, IEEE, 8(9), 1223-1225 (1996).

[5] O. Hadeler, E. Rønnekleiv, M. Ibsen et al., "Polarimetric distributed feedback fiber laser sensor for simultaneous strain and temperature measurements," Applied optics, 38(10), 1953-1958 (1999).

[6] L.-Y. Shao, X. Dong, A. P. Zhang et al., "High-resolution strain and temperature sensor based on distributed Bragg reflector fiber laser," Photonics Technology Letters, IEEE, 19(20), 1598-1600 (2007).

[7] L. Rodríguez Cobo, M. Á. Quintela Incera, S. Rota Rodrigo et al., "Single-longitudinal mode laser structure based on a very narrow filtering technique," (2013). 